\title{
Características presentes en las personas profesionales en Orientación para la promoción del involucramiento del estudiantado
}

Characteristics that School counselors have in order to promote student engagement

\section{Volumen 21, Número 3 \\ Setiembre - Diciembre \\ pp. 1-26}

\author{
Yesennia Céspedes Gamboa \\ Maritza Jara Solano \\ Ramón Ocón Castillo \\ José Pablo Ortiz Monge \\ Carlos David Rodríguez Porras \\ María José Santamaría Sánchez
}

Citar este documento según modelo APA

Céspedes Gamboa, Yesennia., Jara Solano, Martiza., Ocón Castillo, Ramón., Ortiz Monge, José Pablo., Rodríguez Porras, Carlos David., y Santamaría Sánchez, María José. (2021). Características presentes en las personas profesionales en Orientación para la promoción del involucramiento del estudiantado. Revista Actualidades Investigativas en Educación, 21(3), 1-26. Doi. 10.15517/aie.v21i3.46222 


\title{
Características presentes en las personas profesionales en Orientación para la promoción del involucramiento del estudiantado \\ Characteristics that School counselors have in order to promote student engagement
}

\author{
Yesennia Céspedes Gamboa ${ }^{1}$ \\ Maritza Jara Solano \\ Ramón Ocón Castillo \\ José Pablo Ortiz Monge \\ Carlos David Rodríguez Porras \\ María José Santamaría Sánchez
}

\begin{abstract}
Resumen. El presente artículo se desprende del Trabajo Final de Graduación para optar por el grado de Licenciatura en Ciencias de la Educación con énfasis en Orientación de la Universidad de Costa Rica, el cual se realizó desde agosto de 2018 y finalizó en el mes de julio del año 2020. En este estudio se pretende reconocer, a partir de los aportes de las personas participantes, las características que fomentan el involucramiento del estudiantado en su proceso educativo. En la revisión documental no se encontraron estudios donde se enfatizará en las características de las personas profesionales en dicha disciplina. De allí la importancia de la investigación realizada. La metodología correspondió a un enfoque cualitativo y método fenomenológico, se realizaron entrevistas semiestructuradas a profesionales en Orientación en ejercicio y se aplicaron grupos focales con estudiantes de secundaria. Como hallazgos, se obtuvo que las características de apertura, empatía, autenticidad y proactividad enriquecieron el trato digno hacia el estudiantado, lo que favoreció el involucramiento. A su vez, la puesta en práctica de dichas características condujo a la satisfacción profesional de las personas orientadoras. Finalmente, las conclusiones reflejan que las características antes mencionadas permitieron considerar las diferencias entre el estudiantado, además de fomentar el papel protagónico para el involucramiento en su proceso educativo. De igual manera, se concluye que la puesta en práctica de estas características; generó bienestar y satisfacción laboral en las personas profesionales participantes en la investigación.
\end{abstract}

Palabras clave: aprendizaje, educación, enseñanza, estudiante, orientación.

Abstract. This article is derived from the Graduation Final Project to opt for the degree of Licenciatura in Educational Sciences with an emphasis in Counseling from Universidad de Costa Rica, which was performed since August 2018 and finished in July 2020. Based on the contributions of participants, this study aims to recognize their role in the promotion of student engagement in the educational process. Previous studies that specify the characteristics of school counselors for the promotion of student engagement were not found, hence the importance of recognizing this information. The methodology corresponds to a qualitative approach and a phenomenological method; semi-structured interviews are conducted with practicing counseling professionals and focus groups are applied with high school students. It was found that the characteristics of openness, empathy, authenticity, and proactivity enrich their belief in treating students with dignity, which at the same time favors engagement. Also, the implementation of these characteristics leads to counselors' professional satisfaction. Finally, it is concluded that the aforementioned characteristics allow to consider students' individual differences and promote a self-led role for their engagement in the educational process; in the same way, it is concluded that the implementation of these characteristics generates well-being and work satisfaction to the professional person.

Key words: learning, education, student, teaching, counselling.

\footnotetext{
1 La información de las personas autoras aparece al final del artículo
}

Dirección electrónica de contacto: santamaria2096@gmail.com

Artículo recibido: 16 de marzo, 2021

Enviado a corrección: 04 de junio, 2021

Aprobado: 16 de agosto, 2021 


\section{Introducción}

Este artículo se desprende de la realización de un Trabajo Final de Graduación (TFG) para obtener el grado de Licenciatura en Ciencias de la Educación con énfasis en Orientación de la Universidad de Costa Rica, efectuado por Céspedes et al. (2020). El cuestionamiento que atañe al presente artículo hace referencia a reconocer cuál es el rol de las personas profesionales en Orientación en la promoción del involucramiento del estudiantado en el proceso educativo, haciendo énfasis en las características de las personas profesionales desde los aportes de las personas participantes del estudio, quienes fueron profesionales en orientación y estudiantes.

Lo anterior debido a que las personas profesionales en Orientación trabajan junto con estudiantes con el fin de ofrecer acompañamiento y guía, por lo tanto, se profundiza en el rol de las personas profesionales en cuanto al involucramiento del estudiantado. La importancia radica en que, si bien hay funciones específicas asignadas para las personas profesionales en Orientación que laboran en el Ministerio de Educación Pública (en adelante MEP), en este caso se hace énfasis propiamente en las características laborales deseables de las personas profesionales en esta disciplina para la promoción del involucramiento.

En este sentido, existen características que las personas profesionales en Orientación recalcan como relevantes para la promoción del involucramiento del estudiantado de secundaria. Se visualiza a esta persona profesional como promotora y mediadora en este proceso, así como un agente educativo que propicia que la estancia en la institución sea de mayor provecho y satisfacción para las educandas y los educandos.

Es así como se brinda un aporte a la educación costarricense y a la disciplina de la Orientación, debido a que el involucramiento, que se constituye por los componentes cognitivo, afectivo y conductual, tiene influencia en la motivación y participación activa de la persona estudiante en su proceso educativo. Asimismo, se reconoce la necesidad de investigar más acerca de este tema, lo cual se constata en los antecedentes que se mencionan a continuación.

Entre los antecedentes, a nivel internacional, Carranza y Sandoval (2015) evidencian que el personal docente tiene gran influencia en el estudiantado, específicamente en su avance académico y en la creación de convivencia armónica que favorece la participación y preparación para transformar la vida y el entorno. Los mismos autores llegan a la conclusión de que se visualiza al personal docente como un agente primordial para fomentar el 
involucramiento mediante la creación de un ambiente adecuado para fortalecer la permanencia y el sentido de pertenencia.

Aunado a lo anterior, Acevedo, Valenti y Aguiñaga (2017) realizan la investigación titulada "Gestión institucional, involucramiento docente y de padres de familia en escuelas públicas de México", para determinar el efecto del involucramiento docente, de las familias y de la gestión institucional, en el logro escolar del estudiantado. Se concluye que cuanto más alto sea el interés de la familia mayor es el involucramiento docente, lo cual se relaciona con el rol asesor de la persona profesional en Orientación para la promoción del involucramiento.

En lo que respecta al ámbito costarricense, se encuentran antecedentes como el de Ovares (2012), quien abarca la temática de los factores que se relacionan con la exclusión educativa, así como estrategias que utiliza el personal docente y administrativo para la prevención de la problemática, tales como la coordinación interinstitucional y el trabajo interdisciplinario. Otro estudio es el de Guardado y Hernández (2010) acerca del plan estratégico operativo en educación y las principales acciones para la permanencia y éxito escolar; en esta se determina cuáles estrategias de calidad y pertinentes colaboran con las necesidades del estudiantado.

También, se encuentra el estudio cualitativo de Arguedas (2011) realizado desde la disciplina de la Orientación, este trata acerca de recursos docentes para favorecer el involucramiento de estudiantes. Entre los hallazgos se afirma que es esencial que el personal docente posea agrado y respeto hacia la población estudiantil y que reconozca esfuerzos y logros. Aunque es una investigación realizada a nivel nacional, se enfocó en el trabajo docente, por lo que se consideró pertinente obtener la perspectiva de orientadoras y orientadores.

A partir de los estudios consultados, se visualiza un gran aporte de la temática del involucramiento con diferentes poblaciones. Sin embargo, se evidencia que no existe información sobre el involucramiento que pueden fomentar las personas profesionales en Orientación. Este artículo tiene por objetivo el reconocer a partir de los aportes de las personas participantes, las características para la promoción del involucramiento del estudiantado de secundaria en su proceso educativo. 


\section{Referente teórico}

Seguidamente se describen los elementos fundamentales de este estudio a nivel teórico, los cuales le brindan sustento a la investigación como parte esencial del desarrollo del conocimiento en la disciplina de la Orientación. Es de importancia hacer referencia al involucramiento del estudiante en su proceso educativo, el cual es definido por Arguedas (2010) como:

las actitudes de las estudiantes y los estudiantes hacia el colegio, sus relaciones interpersonales dentro de la institución y su disposición hacia el aprendizaje. Se expresa mediante el sentimiento de conexión y la participación en las diversas actividades escolares. Implica que las alumnas y los alumnos participen en actividades académicas y no académicas, que tengan amistades en el colegio y buenas relaciones con docentes, que desarrollen un sentido de pertenencia y que se identifiquen con la institución y los valores por los que esta se rige. (p. 64)

Por su parte, Fredricks, Blumenfeld y Paris (2004) indican que el involucramiento está conformado por tres componentes. El primero es el componente cognitivo, que se relaciona con el esfuerzo mental enfocado en adquirir habilidades y aprendizajes necesarios, así como invertir el tiempo necesario para alcanzar metas educativas de forma autónoma.

El segundo componente es el afectivo, el cual favorece la generación de vínculos con la institución donde se estudia y todos con los agentes educativos; también, son todas aquellas actitudes, intereses, valores y reacciones positivas, que promueven la voluntad para lograr obtener logros educativos y superar tareas de la etapa del desarrollo.

El tercer componente es el conductual, este incluye conductas como cumplir las normas, tener comportamientos relacionados con la persistencia, el esfuerzo, la concentración, la contribución a la discusión en clase, la atención y participación en el aula, así como la ausencia de acciones que puedan representar un obstáculo para el proceso educativo. Además, este tercer componente se relaciona con la participación en actividades extracurriculares.

Por otra parte, para efectos de este artículo es necesario detallar y enmarcar las definiciones alrededor de las características de apertura, de empatía, de autenticidad y de proactividad de las personas profesionales en Orientación para la atención de la población estudiantil en su proceso educativo. Ríos (2009) menciona que las características personales 
y profesionales facilitan el crear acciones que generen cambios necesarios en los centros educativos para mejorar las prácticas educativas.

En este sentido, se conceptualiza el trato digno que se le brinda a la población estudiantil mediante distintos abordajes, de acuerdo con Pérez y Pezoa (2013) significa apoyar sin discriminación alguna, respetando el derecho de las personas a ser tratadas de manera decente, a través de una visión integral de esta; ser escuchada o escuchado, a ser llamada por su nombre, recibir un saludo cordial, tener respeto a su privacidad, y brindar compresión.

Además, para esta investigación se considera importante describir la característica de la apertura, para los autores Corey y Corey (1977) es una característica personal y profesional que se relaciona con la disposición de escuchar a estudiantes, familias y otras personas profesionales de su equipo de trabajo que así lo soliciten o se identifique que requieren la atención; asimismo aceptar y respetar la diversidad de las demás personas.

Aunado a lo anterior, se toma en cuenta la empatía que, de acuerdo con Kottler y Balkin (2017), es el enlace que favorece el entendimiento de la realidad entre seres humanos, fortalece las relaciones interpersonales por medio del reconocimiento del marco de referencia interno del individuo, y así transmitir lo que se comprende, esto facilita la creación de vínculos profesionales entre la persona orientadora y las personas orientadas.

También es necesario considerar la autenticidad, que Vaquer, Carrero, y García-Gaete (2008) definen como la manifestación de la identidad que trasciende el proceso de integración personal, favoreciendo el poder expresarse y autorrealizarse. La autenticidad es una característica vital de una persona profesional en su rol en las instituciones educativas, al permitirle el acercamiento al estudiantado, generando confianza en este.

Por otra parte, se considera importante definir la satisfacción profesional, debido a que tiene una relación recíproca con el desempeño laboral, de acuerdo con Sánchez y García (2017) es una actitud o conjunto de actitudes desarrollados por la persona hacia su trabajo. Es decir, que el desempeño con el que una persona profesional realice su trabajo se ve directamente ligado con la actitud con que realice el mismo. Adicionalmente, de acuerdo con Abrajan, Contreras y Montoya (2009) la satisfacción laboral es ampliamente determinada por la interacción entre el personal y las características del ambiente o el contexto laboral.

Estos autores también toman lo propuesto por Staw y Ross (1985) con respecto a que la satisfacción es la concordancia entre la persona y su puesto; de la misma manera Ruvalcaba, Selva y Sahagún (2014) mencionan a Muchinsky (1994) quien establece y 
distingue la satisfacción profesional como una respuesta afectiva y emocional, y que el afecto hace referencia a una sensación de gusto o disgusto.

Es así como se determina que el desarrollo profesional es necesario para favorecer la relación de la persona profesional en Orientación con su labor en el centro educativo, por ejemplo para promover la adquisición de aprendizajes significativos y atender necesidades específicas de la comunidad educativa y demás acciones propias de acuerdo al MEP, lo cual genera una sensación de disfrute de su quehacer y valoración de sus acciones en su contexto de trabajo, lo que mejora su proactividad y la toma de decisiones para la promoción del involucramiento de sus estudiantes en su proceso educativo.

Como parte de la satisfacción profesional, se desprende la proactividad como un aspecto importante que la complementa, de acuerdo con Ares (2004) citado en Murillo (2017) es una capacidad, donde las personas pueden reconocer la influencia del medio sin determinarlas, en otras palabras, las personas actúan según su voluntad sin dejarse llevar por las influencias del medio.

Las personas proactivas han logrado desarrollar esta característica desde la experiencia, ya que como profesionales en Orientación se puede favorecer el desarrollo de esta capacidad en los entornos laborales. Según Murillo (2017), la proactividad brinda a las personas protagonismo, que les permite gestionar su trabajo de forma autónoma y siempre en función de aprovechar la situación al máximo.

De acuerdo con López (2010), una persona profesional proactiva debe edificar una serie de características que le faciliten desenvolverse en su contexto, como lo son creación de oportunidades, iniciativa, promoción de circunstancias, anticipación de problemas y actitud de control.

Según lo expuesto teóricamente, se ha hecho referencia a esas características en específico debido a que son las que resaltan de acuerdo con las personas profesionales en Orientación participantes de la investigación, esto vinculado al rol que se cumple en secundaria para promover el involucramiento estudiantil.

\section{Metodología}

En este apartado se detalla el proceso metodológico utilizado en la investigación, la cual inició en agosto del año 2018 y finalizó en el mes de julio del año 2020. A continuación, se hace referencia al enfoque, a la población participante, a las técnicas de recolección y procesamiento de la información. 


\subsection{Enfoque}

En primera instancia, la investigación se realiza utilizando un enfoque cualitativo, el cual según Barrantes (2014) y Villalobos (2017) parte de un contexto en específico para investigar un fenómeno, por lo que se describen las relaciones y conductas en torno al mismo, y se interpreta lo captado activamente, dado que se utilizan técnicas de comprensión personal, sentido común e introspección.

En la investigación, este enfoque permitió, tal y como afirma Ruíz (2012), un grado de flexibilidad conforme se llevó a cabo el proceso, además se basó en el método fenomenológico, mismo que facilitó interpretar el significado que las personas profesionales en Orientación le brindan a sus acciones para favorecer el involucramiento, así como las características que consideran esenciales para promoverlo, dado que, en este método, según Barrantes (2014), existen diferentes perspectivas y significados alrededor de las realidades, de acuerdo con experiencias de las personas participantes de una investigación.

\subsection{Población participante}

En lo que respecta a la población participante, primero se tomaron en cuenta a profesionales en Orientación en ejercicio (Ver Tabla 1), con los criterios de inclusión de que: tuvieran como mínimo cuatro años de experiencia laborando en instituciones públicas educativas de secundaria; también, que los centros educativos estuvieron ubicados geográficamente en la gran área metropolitana, principalmente cerca de San José, Costa Rica; tener participación de ambos sexos, con tres hombres y tres mujeres.

En la investigación toma interés la promoción del involucramiento del estudiantado de secundaria, ya que si bien en la primaria es relevante que cada estudiante sea protagonista en su proceso; es en la etapa de la adolescencia donde se refuerza la necesidad de que la persona se responsabilice de su propio desarrollo educativo, social y personal.

\section{Tabla 1}

Datos de las personas profesionales en Orientación participantes

\begin{tabular}{|c|c|} 
Profesional en Orientación & $\begin{array}{c}\text { Años de laborar como profesional en } \\
\text { Orientación en el sistema educativo }\end{array}$ \\
\hline 1. orientador & 25 años \\
\hline 3. orientadora & 21 años \\
\hline 4. orientador & 14 años \\
\hline 5. orientadora & 12 años \\
\hline 6. orientadora & 11 años \\
\hline
\end{tabular}

Fuente: Elaboración propia 
Las personas profesionales en Orientación que participaron en esta investigación laboran en instituciones educativas de distintas modalidades de acuerdo con la oferta educativa del Ministerio de Educación Pública (MEP) para III ciclo y educación diversificada. Las modalidades son las siguientes: tres colegios académicos diurnos, entre ellos Liceo Mauro Fernández Acuña, Colegio de Cedros, Liceo de Pavas y Liceo Edgar Cervantes; una institución de Educación General Básica (IEGB) nombrada Andrés Bello López, que en secundaria ofrece III Ciclo; y un colegio técnico profesional diurno llamado Colegio Técnico Profesional Abelardo Bonilla Baldares en Moravia.

La otra población participante que se incluyó en este estudio fueron 24 estudiantes de secundaria (ver tabla 2) en la etapa de la adolescencia y entre los criterios de selección, se propició que existiera representación de los diferentes niveles desde sétimo a undécimo año; además, se les solicitó consentimiento de las personas encargadas de cada menor de edad y su respectivo asentimiento. Por otra parte, el criterio de inclusión que se planteó, para su participación, fue tomado en cuenta por la recomendación que las personas profesionales entrevistadas hicieron al grupo investigador, ante esto se realizaron dos grupos focales en dos instituciones diferentes, el primero integrado por once estudiantes y el segundo por trece, de acuerdo con Martínez (2012) se toma en cuenta un promedio de 12 personas, quien recomienda esta distribución de participantes para la aplicación de los grupos focales.

Cabe destacar que tanto los grupos focales y las personas profesionales en Orientación se seleccionaron a conveniencia bajo el muestreo intencional, según McMillan y Schumacher (2008) es el más utilizado en la Educación, pues consiste en "tomar sujetos accesibles que puedan representar ciertos tipos de características" (p.147).

Tabla 2

Datos de los Grupos Focales

\begin{tabular}{|c|c|c|c|c|}
\hline $\begin{array}{l}\text { Grupo } \\
\text { Focal }\end{array}$ & $\begin{array}{l}\text { Institución donde se } \\
\text { desarrolló }\end{array}$ & $\begin{array}{c}\text { Cantidad de } \\
\text { hombres }\end{array}$ & $\begin{array}{l}\text { Cantidad de } \\
\text { mujeres }\end{array}$ & $\begin{array}{c}\text { Total de } \\
\text { estudiantes } \\
\text { participantes }\end{array}$ \\
\hline No. 1 & Colegio de Cedros & 6 & 5 & 11 \\
\hline No. 2 & $\begin{array}{l}\text { Liceo Mauro Fernández } \\
\text { Acuña }\end{array}$ & 5 & 8 & 12 \\
\hline
\end{tabular}

Fuente: Elaboración propia

\subsection{Técnicas de recolección}

Referente a las técnicas de recolección de información, en primera instancia se utilizó la entrevista semiestructurada de preguntas abiertas (Anexo 1), dirigida a seis personas profesionales en Orientación de distintas instituciones educativas de secundaria. Hubo dos 
encuentros, en el inicial se plantearon 15 preguntas sobre el involucramiento y sus manifestaciones de acuerdo con los componentes; también se realizaron entrevistas de verificación para clarificar y ampliar aspectos identificados en el análisis de las primeras respuestas obtenidas.

La entrevista semiestructurada de acuerdo con Hernández, Fernández y Baptista (2010), consiste en "una reunión para conversar e intercambiar información entre una persona (el entrevistador) y otra (el entrevistado) u otras (entrevistados)" (p. 418). Por lo cual, se realizan preguntas abiertas que permitan a la persona entrevistada expresar sus experiencias sobre la temática.

Retomando lo expuesto anteriormente en la población participante, se realizaron dos grupos focales en dos de estas instituciones, en los que se incluyeron 15 preguntas abiertas para indagar sobre las acciones que realizan las personas profesionales en Orientación para la promoción del involucramiento y de esta manera contemplar la perspectiva del estudiantado, lo cual permitió triangular la información obtenida, según Hernández et al. (2010), se busca confirmar o corroborar resultados y efectuar validación cruzada entre datos, mediante técnicas de recolección, informantes, investigadores y teoría.

Estas técnicas de recolección de la información, tanto el grupo focal como la entrevista semiestructurada; permitieron indagar el involucramiento como un fenómeno, que es parte de la vida educativa y que implica el desarrollo integral de la persona estudiante desde los componentes afectivos, conductual y cognitivo; reconociendo el rol que tiene la persona profesional en Orientación en su promoción; así mismo facilitó la comprensión de las características que se deben implementar desde la Orientación para poder promoverlo.

De esta manera, la información recolectada en ambas técnicas se analiza tomando como base los objetivos planteados y la teoría de la investigación.

\subsection{Procesamiento de análisis}

El proceso de análisis se llevó a cabo tomando como base a Taylor y Bogdan (1998), de manera que se realizó una primera fase de recolectar información, transcribir y organizar la misma. En la segunda fase se codificó, para Hernández et al. (2010), primero se deben codificar las frases o citas de las entrevistas, lo cual consiste en agruparlas por temas, procurando además que exista un equilibrio entre lo mencionado por todas las personas participantes. Esto facilita encontrar similitudes y contradicciones entre la información que se obtuvo de las personas profesionales en Orientación y el estudiantado. Para efectos de esta 
investigación se asignan diferentes códigos para las personas participantes que comprenden abreviaturas y numeración, por lo que se utilizan para las personas profesionales el código PPO del 1 al 6 y los códigos del GF1 o GF2 para los grupos focales, seguidos por el número de estudiante participante; lo cual se refleja en la Tabla 3.

Tabla 3

Códigos asignados a las personas participantes

\begin{tabular}{|c|l|c|}
\hline Código & \multicolumn{1}{|c|}{ Significado } & Ejemplo \\
\hline PPO\#, H & $\begin{array}{l}\text { Persona Profesional, número } \\
\text { de entrevista, Hombre. }\end{array}$ & PPO6, M \\
\hline PPO\#, M & $\begin{array}{l}\text { Persona Profesional, número } \\
\text { de entrevistada, Mujer. }\end{array}$ & GF1, E11, H, U \\
\hline GF\#, E\#, H, N & $\begin{array}{l}\text { Grupo focal, número de } \\
\text { estudiante, Hombre, nivel. }\end{array}$ & GF2, E2, M, S \\
\hline GF\#, E\#, M, N & $\begin{array}{l}\text { Grupo Focal, número de } \\
\text { estudiante, mujer, nivel. }\end{array}$ \\
\hline
\end{tabular}

Fuente: Elaboración propia

En la tercera fase se interpretaron los datos, la información se dirige a conformar dimensiones, categorías y subcategorías (Ver figura 1), las cuales se llevaron a cabo entre el grupo investigador y personas integrantes del comité asesor, tomando en cuenta la fundamentación teórica.

Figura 1

Representación de la red de dimensión 2: Categorías y subcategorías

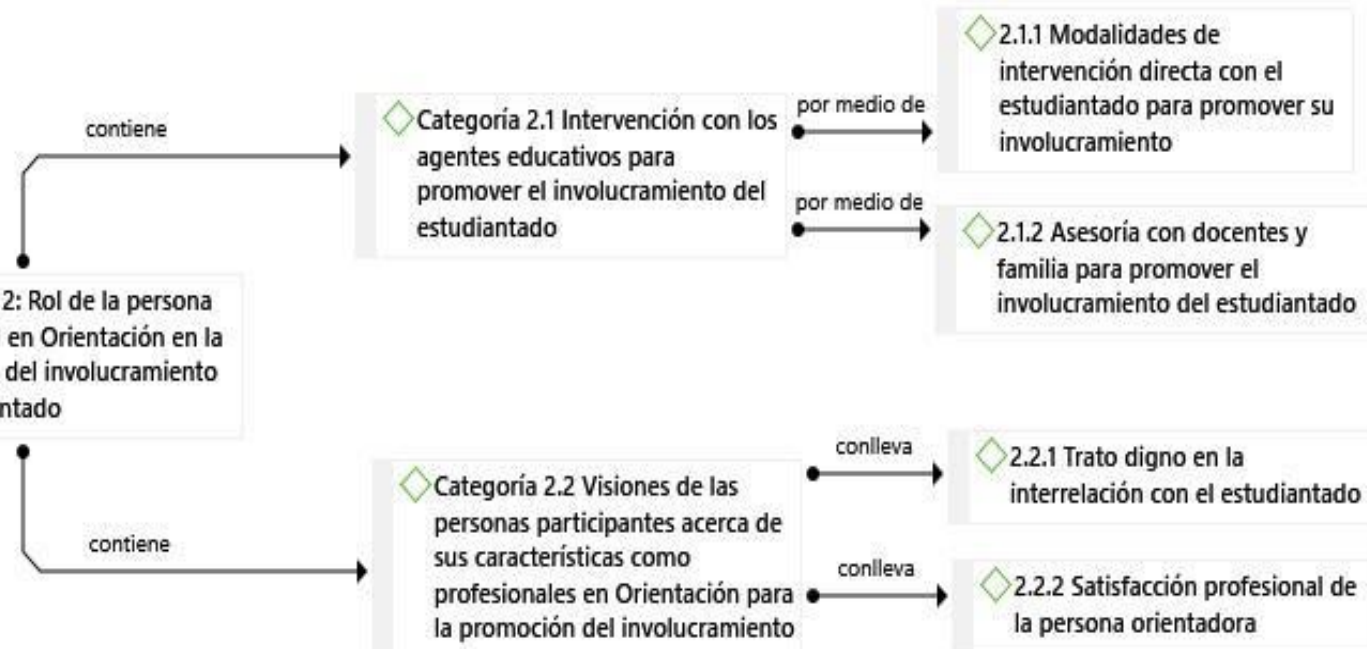

Fuente: Céspedes et al (2020). 
Para realizar la construcción de estas categorías de análisis, se utilizó la herramienta Atlas.ti que, según Lewis (2004) y Hwang (2007), es un programa de análisis cualitativo asistido por computadora para manejar la información en diferentes sentidos, como: asociar códigos o etiquetas con fragmentos de texto, sonidos, imágenes, en formato digital; además se buscan patrones de códigos y esto se clasifica en categorías de análisis.

Esta herramienta, específicamente la Versión 8.0, apoyó la interpretación por parte de las personas investigadoras y el uso de procedimientos sistemáticos, así como la flexibilidad en la revisión de los procesos de análisis.

\section{Resultados}

A continuación, se profundiza en las características de la persona profesional en Orientación para la promoción del involucramiento. En la Figura 2, se presentan dichas características, además permite visualizar como el trato digno influye en cada una de estas, al promover el involucramiento.

Figura 2

Características para la promoción del involucramiento

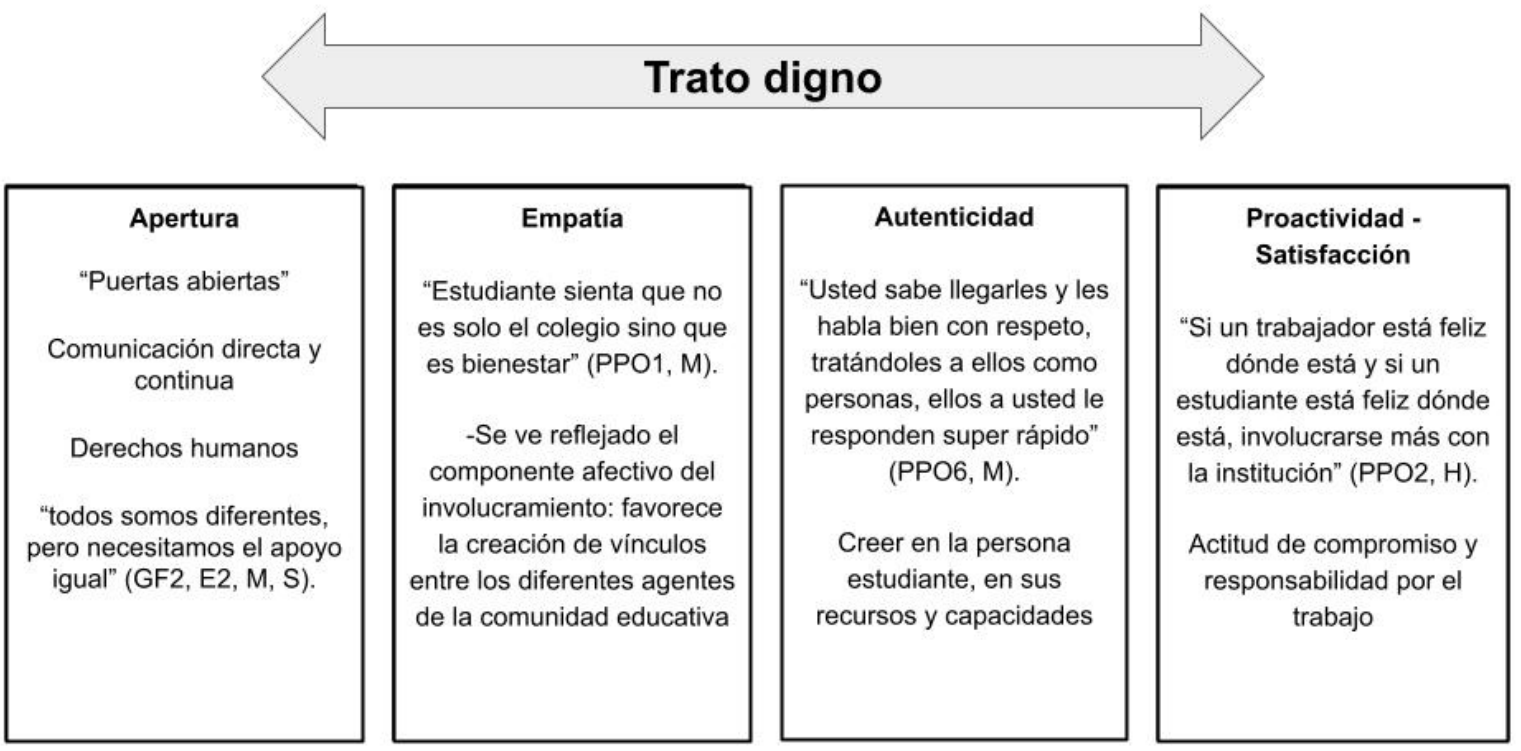

Fuente: Elaboración propia.

Las características de la persona profesional en Orientación para la promoción del involucramiento, dentro del quehacer diario en sus respectivas funciones, como orientar, asesorar y planificar, son variadas según la situación específica que se aborde. A través de la investigación llevada a cabo, se identificaron características como la empatía, apertura, 
autenticidad, proactividad, que permiten promover el involucramiento en el estudiantado de secundaria, las cuales se llevan a la práctica por medio del trato digno en la interrelación con el estudiantado y la satisfacción profesional de la persona orientadora en su rol. A partir de este punto, se desarrolla cada característica y cómo se interrelacionan para la promoción de los componentes que favorecen el involucramiento.

Como parte esencial para favorecer el involucramiento se encuentra el trato digno en la relación con la persona estudiante, de manera humanista y respetuosa; este aspecto involucra características tales como la apertura, la empatía, la autenticidad y la proactividad, las cuales son destacadas reiteradamente por las personas profesionales en Orientación entrevistadas.

Las orientadoras y los orientadores participantes del estudio coinciden en la frase "puertas abiertas", la cual se relaciona con la característica de la apertura que se le debe transmitir a las personas estudiantes, permitiendo una comunicación directa y continua, lo que refuerza ese trato digno con el estudiantado en medio de las funciones que tiene la persona profesional de la disciplina ya mencionada. Maños (1998) afirma que de dicha relación se puede favorecer estímulos que facilitan procesos que buscan atender las necesidades de la persona estudiante.

En relación con lo anterior, se rescata lo afirmado por Pérez y Pezoa (2013) con respecto a que toda persona tiene derecho a ser llamada por el nombre, recibir saludo, comprensión, ser escuchada y no recibir discriminación, por lo que en frases de las personas profesionales en Orientación, se refleja la apertura y la empatía que, forman parte de la relación con el trato digno entre persona orientadora y el estudiantado, lo cual se evidencia en el siguiente aporte de una persona profesional en Orientación:

"que el estudiante sienta que no es solo el colegio sino que es bienestar, o sea, yo puedo decirle a mi profesor, a mi orientador, mire es que me está pasando esto y esto y en el colegio no solo me van a enseñar de materias, sino que también me van a responder con esa otra parte, esa creo que es la característica más importante" (PPO1,M)

Por lo tanto, cada alumna y cada alumno puede percibir la empatía y apertura de la persona profesional, lo cual favorece una relación de respeto y colaboración, en la cual se promueve el descubrimiento de destrezas, espacios para que expresen su sentir, decisiones con relación con la institución educativa, informando sobre oportunidades de mejora que 
beneficien su desarrollo, al reconocerlas y reconocerlos como seres independientes e integrales.

En otras palabras, los espacios promovidos desde la disciplina de la Orientación en los centros educativos deben permitir la libre expresión de cada estudiante; destacando el respeto por las diferentes formas de pensar, sentir y actuar. Para fortalecer la autonomía e individualidad en el proceso de aprendizaje cooperativo y desarrollar en la población estudiantil el involucramiento en su propio crecimiento personal y educativo.

Además, el MEP (2016) admite que la comunidad educativa debe fomentar el ejercicio de los derechos humanos, para fortalecer el trato digno y la expresión libre de discriminación y violencia; por lo que la persona profesional en Orientación, mediante la característica de la apertura, puede construir espacios de empatía, respeto y solidaridad entre los diferentes agentes educativos de la institución, tomando en cuenta las vivencias del estudiantado.

De esta manera, se reconoce la empatía como otra de las características resaltadas por las personas orientadoras participantes para fomentar el trato digno, ya que, dentro de sus funciones, les va a permitir sensibilizar al personal docente sobre las diferentes realidades inherentes a la etapa de desarrollo de la persona adolescente. Una de las personas profesionales señala que, con respecto al estudiando, es importante "marcarle sus límites, pero sí tratar de entender las situaciones por las que ellos pasan" (PPO4, H), lo cual concuerda con lo mencionado por López, Filippetti y Richaud (2014), al conceptualizar la empatía como "la capacidad de comprender al otro, basada en el reconocimiento de ese otro" (p. 40).

La empatía contribuye en la promoción del involucramiento del estudiantado, dado que perciben que la persona profesional les escucha, reconoce y se esfuerza por comprender sus sentimientos y perspectivas. En este sentido, mediante el trato digno, se favorecen los componentes del involucramiento del estudiantado que le permita alcanzar un desarrollo y bienestar integral.

Del mismo modo, se ve reflejado el componente afectivo del involucramiento cuando se pone en práctica la característica de la empatía, porque favorece la creación de vínculos entre los diferentes agentes de la comunidad educativa; a su vez permite a cada estudiante sentirse parte de la institución contribuyendo a su sentido de pertenencia.

Aunado a lo anterior, en el grupo focal, las personas estudiantes hacen referencia a cómo distintos profesionales en Orientación toman en cuenta sus emociones y sentimientos en su contexto inmediato y hace lo posible por modificar algunos aspectos para el logro del 
bienestar, lo cual es resaltado por Marchesi (2018), quien señala que la defensa de los derechos humanos y de la dignidad es parte importante de la formación integral del estudiantado en secundaria.

Al respecto de las características mencionadas que favorecen el trato digno, la apertura y la empatía en una relación de ayuda debe tomar en cuenta las particularidades de la población y su contexto, con la finalidad de desarrollar el involucramiento en cada estudiante a través del reconocimiento de su individualidad. Esto se refleja en lo que mencionó una estudiante participante del grupo focal "a pesar de todo, tener en cuenta que todos somos diferentes, pero necesitamos el apoyo igual" (GF2, E2, M, S).

Desde lo expresado por la estudiante en el grupo focal, es necesario reconocer las diferencias culturales, intereses y habilidades particulares de cada estudiante, lo cual enriquece la convivencia en las instituciones a las que asisten. Otro aspecto importante hace referencia a la etapa del desarrollo en la que se encuentra el estudiantado de secundaria; que se centra en la construcción de su identidad. Mediante las características de la empatía y apertura, las personas profesionales crean espacios donde se fomente el desarrollo de la misma.

En este sentido, la disciplina de la Orientación, de acuerdo a lo que establece el MEP (2016), reconoce lo singular en cada persona, desde un enfoque de derechos humanos, y según las necesidades particulares, por lo que es vital que se lleve a la práctica en el ejercicio profesional.

Otra característica de la persona profesional en Orientación que permite dar un trato digno al estudiantado es la autenticidad. Timoneda (2015), al respecto de esta, indica que se demuestra en el hecho de ser coherente con lo que se expresa. De esta manera, cuando la persona profesional en Orientación interviene en procesos, debe dar valor al hecho de creer en la persona estudiante, en sus recursos y capacidades, esto le permite interaccionar desde la característica de la autenticidad y de los diferentes componentes del involucramiento.

Se complementa con lo que una profesional en Orientación comparte:

"trabajar con los chicos es muy fácil, hay chicos que son más complicados, pero si usted sabe llegarles y les habla bien con respeto, tratándoles a ellos como personas, ellos a usted le responden super rápido y le responden bien" (PPO6, M). 
La persona profesional de la Orientación, al poner en práctica las diferentes características que emergieron como la apertura, empatía y autenticidad en su quehacer, facilita el involucramiento de cada estudiante en su proceso educativo y una atención basada en el trato digno, en esta investigación ambos se consideraron como primordiales para tales fines desde la perspectiva de las personas participantes.

Lo anterior debido a que el involucramiento de la persona estudiante al estar constituido por los elementos cognitivos, afectivos y conductuales, las personas que les rodean, entre estas, profesionales en Orientación, pueden favorecer experiencias y conocimientos que influyen en los aprendizajes, esfuerzos, aspectos de mejora al momento de estudiar, incorporación de distintas estrategias de estudio, identificación y manejo de emociones, reafirmación de intereses vocacionales, entre otras.

Aunado a lo anterior, según las personas profesionales en Orientación participantes de esta investigación, la satisfacción de cumplir con la labor profesional le permite promover el involucramiento, ya que la empatía, apertura, autenticidad y proactividad, tienen un impacto en su quehacer profesional, tal y como lo explica la profesional a continuación

"al final queda la satisfacción cuando uno ve los resultados, ver los cambios que han tenido mis chicos desde sétimo hasta ahorita en décimo y sé que ellos van a seguir creciendo, y aplicando lo que se aprende en su vida cotidiana, a veces llegan los padres y dicen "waao, qué cambio más grande", eso para mí es muy satisfactorio y es lo que le da energía y una satisfacción personal y profesional” (PPO3, H).

De esta manera, referente a la satisfacción profesional, Apuy (2008), citando a Davis (1991) afirma que esta es resultado de la percepción que se tiene de su propio trabajo, se debe considerar el ambiente de la institución, las actividades que se realizan, la interacción con otras personas, el acatamiento de reglas, el logro del rendimiento y la remuneración.

Cabe mencionar que, al ser la satisfacción profesional reiterada por las personas profesionales participantes, es posible mencionar que este tópico es significativo e influyente en la labor que realizan las personas profesionales en Orientación, lo cual puede significar que el sentirse a gusto con su trabajo puede estar en relación con aspectos como el clima institucional, elección vocacional, habilidades para enfrentar el mundo laboral y posibilidades de cumplir con objetivos propuestos.

En concordancia con este aporte, un orientador entrevistado menciona: "si un trabajador está feliz dónde está y si un estudiante está feliz dónde está, engancharse más, 
involucrarse más con la institución" (PPO2, H). De este modo, este sentimiento le permite a la persona profesional generar interés por las necesidades del estudiantado y promover el involucramiento de estos con su proceso educativo.

En la misma línea, una de las profesionales afirma que, "hay mucha necesidad, para mí Orientación es clave, es trascendental en nuestros sistemas educativos, es el mediador entre el estudiante y el docente, no dando quejas, sino viendo cómo los pueden ayudar" (PPO5, M). Por lo que colaborar con el estudiantado provoca satisfacción profesional en las personas participantes, al hacer su trabajo y sentirse útiles dentro de la organización educativa, lo cual es vital para brindar acompañamiento en los procesos educativos, personales, familiares y sociales de las alumnas y los alumnos que estén a su cargo.

Adicionalmente, Murillo (2015) afirma que la satisfacción profesional está compuesta por elementos internos como también externos del mismo ser humano; con respecto a lo interno son las propias características de las personas; por lo que es importante generar espacios de reflexión propia sobre la labor que se realiza en la institución educativa, considerando aspectos positivos y de mejora que favorezcan la satisfacción profesional.

Aunado a lo anterior, los elementos externos, como actividades para la comunidad docente donde se incluyan a todas las personas profesionales en la disciplina de la Orientación, permite que se compartan experiencias de aprendizaje, para de esta manera favorecer la pertenencia e involucramiento con su equipo de trabajo, y desarrollar en conjunto características que les permitan brindar la mejor atención al estudiantado, y por ende fomentar la satisfacción profesional.

En este sentido, se evidencia que es significativo que las características profesionales de personas orientadoras son un medio para llevar a cabo su trabajo de calidad en las intervenciones, enfrentando retos y limitaciones, lo que favorece las condiciones para el aprendizaje de cada estudiante con miras a disfrutar y concluir su proceso educativo de secundaria e incorporarse a la sociedad activamente.

Las personas profesionales entrevistadas, mantienen una actitud de compromiso y responsabilidad por su trabajo, que creen y confían en la importancia de este, que se desenvuelven de forma proactiva, logrando identificar resultados positivos en su centro educativo para el cual ofrece sus servicios profesionales, involucrando a toda la comunidad educativa.

Asimismo, se rescata lo mencionado por Arguedas (2011) quien afirma que la valoración del trabajo y la preparación para el mismo, son necesarias para fomentar el 
involucramiento, ya que el estudiantado percibe la dedicación y el tiempo que se invierte, de manera que muestran interés, por ello es fundamental un conocimiento amplio, en cada temática que se busca enseñar, además, de compromiso para mejorar cada día en la práctica profesional, evaluando cada acción que se realiza y consultando por sugerencias a las personas orientadas.

En relación con lo anterior, se resalta una correlación entre la proactividad y la satisfacción de la persona profesional en Orientación, por lo que entre más se mejoren las intervenciones que lleve a cabo, mayor será su bienestar y el de la comunidad educativa.

También, el involucramiento del estudiantado se ve directamente beneficiado por la satisfacción profesional ya que las personas estudiantes pueden percibir el compromiso y esfuerzo con el que la persona profesional en Orientación desarrolle su trabajo independientemente si la modalidad es grupal o individual, así como en asesoría a docentes y familias, cumpliendo con su rol en la promoción del Involucramiento del estudiantado.

En síntesis, las personas profesionales en Orientación deben de mantenerse en constante actualización, tener iniciativa, generar alternativas, así como adaptarse a las condiciones y situaciones. El trato digno es influenciado con el respeto, la escucha, los derechos humanos, ofreciendo servicios de calidad que motiven al desarrollo personal y social.

Por último, generar y mantener las características de empatía, apertura, autenticidad y proactividad, cada vez que se trabaje desde la disciplina de la Orientación, es vital para que a su vez se genere satisfacción laboral, y fomente el trabajo en equipo en todo el centro educativo.

\section{Conclusiones}

A continuación, se presentan los principales aportes del artículo, con respecto a las características esenciales que posee una persona profesional en Orientación para promover el involucramiento. A su vez se contribuye a las acciones que realiza en su labor, recomendando su aplicación en el quehacer profesional.

De esta manera, dentro de las características que tiene una persona profesional en Orientación para promover el involucramiento, se destacan: la empatía, la apertura, la autenticidad y la proactividad; las cuales se relacionan con la satisfacción profesional y su rol laboral, aspectos que conllevan construir un vínculo en su entorno de trabajo, lo que permite posicionar a la disciplina de la Orientación en los centros educativos. 
Las características mencionadas facilitan brindar un trato digno al estudiantado al considerar las diferencias personales, mediante el papel protagónico de cada estudiante. Lo que posibilita el acompañamiento e interacción en la relación profesional para promover el involucramiento mediante el respeto a su pensar y sentir.

Tanto la empatía, la apertura, la autenticidad y la proactividad influyen en el rol profesional al generar bienestar y satisfacción laboral, derivado de las acciones implementadas con la población estudiantil. El reconocimiento y valoración de las mismas acciones conllevan a la satisfacción por el trabajo realizado y visualizan los aportes de la Orientación en el proceso educativo del estudiantado.

A partir de lo mencionado, se recomienda fortalecer la presencia de la disciplina en la institución educativa y se demuestren las características de la persona profesional para fomentar el trabajo en equipo desde el Departamento de Orientación. Así como planear espacios de participación y acciones través de la apertura, empatía, autenticidad y proactividad, colaboración con los agentes educativos, además, se sugiere para las casas formadoras proponer experiencias en las futuras personas profesionales en Orientación, donde se fortalezcan diferentes características como autenticidad y proactividad. Cabe resaltar que es importante el rol de la persona Orientadora por su dinamismo y función como enlace con las diferentes poblaciones que conforman las instituciones educativas.

Para quienes deseen realizar futuras investigaciones desde la disciplina de la Orientación en el tema del involucramiento, se pueden generar interrogantes como la relación de la familia y personal docentes de las instituciones con el involucramiento, investigar factores institucionales que influyen en la promoción del involucramiento del estudiantado en su proceso educativo, así como investigar acerca de la satisfacción profesional. También, se recomienda realizar estudios sobre el involucramiento y Orientación con diferentes poblaciones como enseñanza primaria, estudiantes de universidades y personas trabajadoras.

Se plantea como un reto a futuro contemplar a la persona profesional en Orientación como promotora del involucramiento en los procesos educativos, lo cual implica generar profesionales que se involucren con su trabajo partiendo desde su formación universitaria. Además, se debe tomar en cuenta el contexto en el cual se promueva el involucramiento y la tecnología como herramienta para superar retos en el aprendizaje. 
Por último, se destaca como posible limitante fue la coordinación de los espacios para la aplicación entrevistas y grupos focales por motivo de horarios laborales, aunque esto no restó fluidez al proceso de investigación.

\section{Agradecimientos}

Se extiende un agradecimiento a profesionales y estudiantes participantes en la investigación. Asimismo, a la Escuela de Orientación y Educación Especial y la Biblioteca de la Facultad de Educación de la Universidad de Costa Rica, por el apoyo brindado durante la implementación del Trabajo Final de Graduación.

\section{Referencias}

Abrajan, María Guadalupe., Contreras, Jose Manuel. y Montoya, Sandra. (2009). Grado de satisfacción laboral y condiciones de trabajo: una exploración cualitativa. Enseñanza e Investigación en Psicología, 14(1),105-118. Recuperado de https://www.redalyc.org/articulo.oa?id=29214108

Acevedo, Carlos., Valenti, Giovanna. y Aguiñaga, Eduardo. (2017). Gestión institucional, involucramiento docente y de padres de familia en Escuelas Públicas de México. Calidad en la educación, (46), 53-95. doi: http://dx.doi.org/10.4067/S0718$\underline{45652017000100053}$

Apuy, Lorena. (2008). Factores del Clima Organizacional que influyen en la Satisfacción Laboral del Personal de Enfermería, en el Servicio de Emergencias del Hospital San Rafael de Alajuela, Junio-Noviembre 2008 (Tesis de Maestría Inédita). Universidad Estatal a Distancia, Costa Rica.

Arguedas, Irma. (2010). Involucramiento de las estudiantes y los estudiantes en el proceso educativo. REICE Revista Iberoamericana sobre Calidad, Eficacia y Cambio en Educación, 8(1), 63-78. Recuperado de https://www.redalyc.org/pdf/551/55113489005.pdf

Arguedas, Irma. (2011). Recursos docentes para favorecer el involucramiento de estudiantes de educación secundaria en el proceso educativo. Revista Iberoamericana de Educación, 2(56), 1-12. doi: https://doi.org/10.35362/rie5621534

Barrantes, Rodrigo. (2014). Investigación: Un camino al conocimiento Un enfoque Cualitativo, Cuantitativo y Mixto ( $2^{a}$ ed.). San José, Costa Rica: Editorial Universidad Estatal a Distancia.

Carranza, Leticia. y Sandoval, Eduardo Andrés. (2015). Docencia, convivencia y permanencia escolar en un Bachillerato Tecnológico. Ra Ximhai, 11(1), 83-108. doi: https://doi.org/10.35197/rx.11.01.2015.05.lc 
Céspedes, Yessenia., Jara, Maritza., Ocón, Ramón., Ortiz, Jose Pablo., Rodríguez, Carlos. y Santamaría, María José. (2020). Rol de la persona profesional en Orientación en la promoción del involucramiento del estudiantado de secundaria en su proceso educativo (Tesis de Licenciatura inédita). Universidad de Costa Rica, Costa Rica.

Corey, Gerald. y Corey, Marianne. (1977). Groups. Process and Practice. Monterey, California: Brooks/Cole.

Fredricks, Jennifer., Blumenfeld, Phyllis. y Paris, Alison. (2004). School Engagement: Potential of the concept, State of the Evidence. Review of Educational Research, 74(1), 59- 109. doi: https://doi.org/10.3102/00346543074001059

Guardado, Yamileth. y Hernández, Karla. (2010). El Plan Estratégico Operativo en Educación y las principales acciones enfocadas para la permanencia y éxito escolar de los y las adolescentes, de centros educativos públicos de secundaria (Tesis de Licenciatura). Universidad de Costa Rica. San José, Costa Rica. Recuperado de http://www.ts.ucr.ac.cr/binarios/tfglic/tfg-l-2010-10.pdf

Hernández, Roberto., Fernández, Carlos. y Baptista, Lucía. (2010). Metodología de la Investigación ( $4^{\mathrm{a}}$ ed.). México DF, México: Mc Graw Hill editores.

Hwang, Sungsoo. (2007). Utilizing qualitative data analysis software: A review of ATLAS.ti. Social Sciene Computer Review 26(4), 519-527. doi: https://doi.org/10.1177/0894439307312485

Kottler, Jeffrey. y Balkin, Richard. (2017). Relationships in Counseling and the Counselor's Life. Virginia, EEUU: American Psychological Association.

Lewis, Barry. (2004). NVivo 2.0 and ATLAS.ti 5.0: A comparative review of two popular qualitative data-analysis programs. Field Methods, 26(4), 439-469. doi: https://doi.org/10.1177/1525822X04269174

López, Alejandra. (2010). La proactividad empresarial como elemento de competitividad. Ra Ximhai: revista científica de sociedad, cultura y desarrollo sostenible, 6(2), 303-312. Recuperado de https://www.redalyc.org/pdf/461/46115146011.pdf

López, Mariana., Filippetti, Vanessa. y Richaud, María Cristina. (2014). Empatía: desde la percepción automática hasta los procesos controlados. Avances en Psicología Latinoamericana, 32(1), 37-5. doi: https://doi.org/10.12804/apl32.1.2014.03

McMillan, James., y Schumacher, Sally. (2008). Investigación Educativa. Madrid, España: Pearson.

Maños, Quico. (1998). La relación de ayuda como técnica interactiva. Educación social Revista de intervención socioeducativa, 10, 10-28. Recuperado de https://www.raco.cat/index.php/EducacioSocial/article/view/175660 
Marchesi, Álvaro. (2018, 24 de abril). Educar en la dignidad de las personas. [Mensaje en un blog]. Recuperado de https://educacionabierta.org/educar-en-la-dignidad-de-laspersonas/

Martínez, Nelson. (2012). Reseña metodológica sobre los grupos focales. Diálogos, 6(9), 4753.

Ministerio de Educación Pública (MEP). (2016). Unidad didáctica para el mes de marzo: Mes de los Derechos Humanos y la lucha contra la discriminación. San José, Costa Rica. Viceministerio Académico. Recuperado de https://mep.go.cr/sites/default/files/page/adjuntos/2-unidad-didactica-mes-derechoshumanos-contra-discriminacion.pdf

Murillo, Osvaldo. (2015). Orientación Laboral: un acompañamiento en el mundo de trabajo. En Alejandrina Mata (Ed.), El desarrollo teórico de la Orientación: un aporte de la Universidad de Costa Rica (pp. 245-289). San José, Costa Rica: Editorial de la Universidad de Costa Rica.

Murillo, Osvaldo. (2017). Los seis pilares de la empleabilidad: Un aporte desde la Orientación Laboral. San José, Costa Rica.

Ovares, Rodolfo. (2012). Análisis de las estrategias para la prevención de la deserción y retención de la población estudiantil que lleva a cabo el personal docente y administrativo del Liceo de Miramar, de la Dirección Regional de Educación de Puntarenas, Costa Rica. Revista Gestión de la Educación, 2(2), 1-27. doi: https://doi.org/10.15517/rge.v2i2.5867

Pérez, Virtudes. y Pezoa, Marcela. (2013). ¿Qué es "trato digno" para los pacientes? Recuperado de http://www.supersalud.gob.cl/difusion/665/articles-9004 recurso 1.pdf

Ríos, Daniel. (2009). Características personales y profesionales de profesores innovadores. Para la construcción del saber, 39(1-2), 153-169. Recuperado de https://www.redalyc.org/pdf/270/27015065007.pdf

Ruíz, José Ignacio. (2012). Metodología de la investigación cualitativa (5a Ed.). Bilbao, España: Universidad de Deusto Bilbao.

Ruvalcaba, Javier., Selva, Clara. y Sahagún, Miguel. (2014). Satisfacción laboral: una revisión teórico-histórica de su investigación. Recuperado de https://www.researchgate.net/publication/298214529 Satisfaccion laboralna revision teorico-historica de su investigacion

Sánchez, Magda Gabriela. y García, Maria De Lourdes. (2017). Satisfacción Laboral en los Entornos de Trabajo. Una exploración cualitativa para su estudio. Scientia Et Technica, 22(2), 161-166. doi: https://doi.org/10.22517/23447214.11471

Staw, Barry. y Ross, Jerry. (1985). Estabilidad en medio del cambio: un enfoque disposicional a las actitudes laborales. Revista de psicología aplicada, 70(3), 469-480. doi: https://doi.org/10.1037/0021-9010.70.3.469 
Taylor, Steven. y Bogdan, Robert. (1998). Introducción a los métodos cualitativos de la investigación. Buenos Aires, Argentina: Ediciones Paidós.

Timoneda, Carme. (2015). Orientando la Orientación: Guía práctica de Orientación Educativa. España: Fundación Carme Vidal Xifre de Neuropsicopedagogia.

Vaquer, Antonio., Carrero, Virginia. y Garcia-Baceteñ Francisco. (2008). Autenticidad del profesorado: implicaciones en el desarrollo de competencias docentes. Recuperado de http://repositori.uji.es/xmlui/bitstream/handle/10234/170751/vaquer 2011 Aut.pdf?seq uence $=3 \&$ is Allowed $=y$

Villalobos, Luis Ricardo. (2017). Enfoques y diseños de Investigación social: cuantitativos, cualitativos y mixtos. San José, Costa Rica: EUNED. 


\section{Anexo}

\section{Anexo 1}

\section{Entrevista semiestructurada a Profesionales en Orientación}

Entrevista semiestructurada dirigida a personas profesionales en Orientación

Este instrumento de recolección de información es parte de una investigación de la Universidad de Costa Rica dirigida a la persona profesional en Orientación que labore en centros educativos de secundaria de la provincia de San José.

Se busca analizar las acciones promotoras del involucramiento del estudiantado de secundaria en su proceso educativo, que realizan las personas profesionales en Orientación. La información que usted brinde es importante para contribuir al conocimiento de la disciplina.

Preguntas iniciales de carácter sociodemográfico:

- Sexo:

- Edad:

- Lugar de trabajo:

- Puesto laboral:

- Experiencia laboral:

- Modalidades de Orientación que trabaja en la institución:

Se da una definición de involucramiento y manifestaciones y se les pregunta por lo que hacen al respecto.

1. ¿Cómo promueve usted como profesional en Orientación, que las relaciones interpersonales en la institución sean favorables?

2. ¿Cómo favorece usted que el estudiantado reconozca sus propios logros?

3. ¿Cómo se fomenta que el estudiantado se sienta parte de su grupo y de la institución educativa?

4. ¿Describa las acciones que se llevan a cabo desde Orientación para que el estudiantado cumpla tareas y normas?

5. ¿Cómo favorece usted la participación del estudiantado en diferentes actividades dentro de la institución?

6. ¿Qué acciones se deben de tomar en cuenta para que el estudiantado persista en sus esfuerzos a lo largo de su proceso educativo?

7. ¿Cómo favorece usted que el estudiantado incorpore una mentalidad de búsqueda de soluciones ante sus retos académicos? 
8. ¿De qué manera promueve usted que el estudiantado analice si sus estrategias para aprender están siendo exitosas?

9. ¿Cómo favorece usted el incremento de la atención y concentración del estudiantado?

10. ¿Cómo ha sido el acompañamiento por su parte para la transición de la escuela al colegio?

11. Viendo la otra parte en este tema, ¿Qué hace usted cuando un estudiante no está enganchado en su proceso educativo?

12. ¿Cuáles acciones se toman para hacer un uso de las TIC para favorecer el involucramiento del estudiantado en su proceso educativo?

13. ¿Qué desafíos encuentra como profesional de la orientación para la promoción del involucramiento en la educación secundaria? 


\section{Información de las personas autoras}

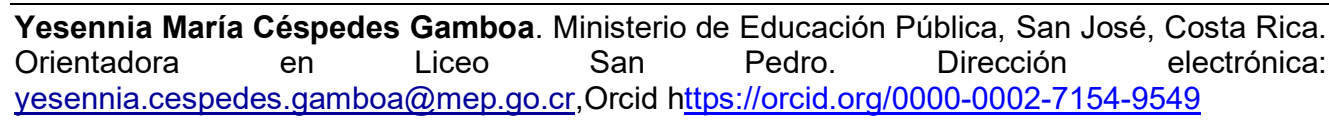

Maritza Jara Solano. Universidad de Costa Rica, San José, Costa Rica. Orientadora del Centro de Asesoría Estudiantil de la Oficina de Orientación. Dirección electrónica: maritza.jara@ucr.ac.cr, Orcid https://orcid.org/0000-0001-9868-8218

Ramón Ocón Castillo. Ministerio de Educación, San José, Costa Rica. Dirección electrónica: ramono.ocon.castillo@mep.go.cr Orcid https://orcid.org/0000-0002-1105-4528

José Pablo Ortiz Monge. Ministerio de Educación, San José, Costa Rica. Orientador en Escuela Junquillo Abajo. Dirección electrónica: joseportiz93@gmail.com, Orcid https://orcid.org/0000-0003-3542-7728

Carlos David Rodríguez Porras. Ministerio de Educación, San José, Costa Rica. Orientador en el Benemérito Liceo José Martí. Dirección electrónica: carlosropo22@gmail.com, Orcid https://orcid.org/0000-0001-8412-5908

María José Santamaría Sánchez. Universidad de Costa Rica, San José, Costa Rica. Asistente de la Unidad de Gestión, Evaluación y Seguimiento de la Calidad - Sistema de Estudios de Posgrado. Dirección electrónica: santamaria2096@gmail.com, Orcid https://orcid.org/0000$\underline{0002-7849-5782}$ 
Revista indizada en

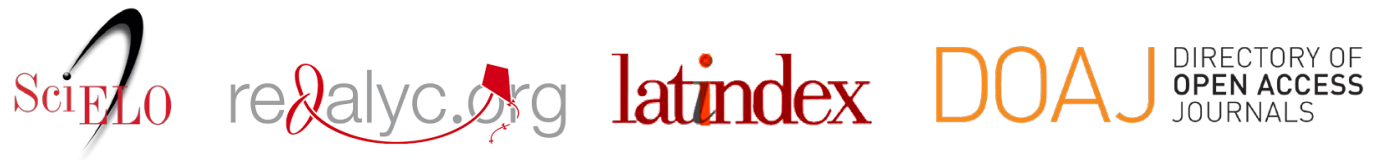

Distribuida en las bases de datos:

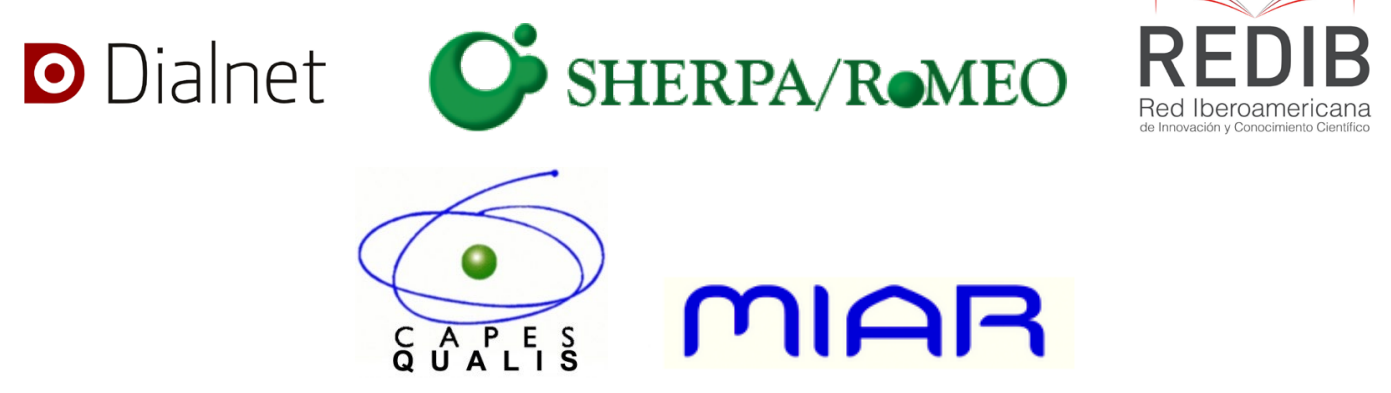

